\title{
Interactions in reentrant phase behavior of a charged nanoparticle solution by multivalent ions
}

\author{
Sugam Kumar, ${ }^{1}$ Indresh Yadav, ${ }^{1,2}$ Sohrab Abbas, ${ }^{1}$ Vinod K. Aswal,,${ }^{1,2, *}$ and Joachim Kohlbrecher ${ }^{3}$ \\ ${ }^{1}$ Solid State Physics Division, Bhabha Atomic Research Centre, Mumbai 400 085, India \\ ${ }^{2}$ Homi Bhabha National Institute, Mumbai 400094, India \\ ${ }^{3}$ Laboratory for Neutron Scattering and Imaging, Paul Scherrer Institut, CH-5232 PSI Villigen, Switzerland
}

(Received 31 July 2017; published 26 December 2017)

\begin{abstract}
The interactions following a reentrant phase transition of charged silica nanoparticles from one phase to two phases and back to one phase by varying the concentration of multivalent counterions have been examined. The observations are far beyond the framework of Debye-Hückel or even nonlinear Poisson-Boltzmann equations and demonstrate the universal behavior of multivalent counterion-driven charge inversion. We show that the interplay of multivalent counterion-induced short-range attraction and long-range electrostatic repulsion between nanoparticles results in reentrant phase behavior.
\end{abstract}

DOI: 10.1103/PhysRevE.96.060602

The understanding of effective interactions dictating the phase behavior of charged colloids is always of interest to achieve a tunable architecture having colloids as building blocks [1-3]. The delicate balance of attractive and repulsive interactions present in the system governs the stability of these colloids. Any manipulation of this interplay of intercolloidal interactions may lead to intriguing phase transformations $[3,4]$. However, in some cases, external physiochemical parameters can be used to tune these interactions such that the system reverts into its initial phase, causing the so-called reentrant phase behavior. Such reentrant phase behavior has been observed in a variety of systems [5-14]. For example, a combination of nonadsorbing polymer-induced depletion interactions and polymer-polymer repulsions is shown to cause reentrant stabilization in nanoparticle-polymer systems [9]. On the contrary, adsorbing polymers are observed to give rise to a reentrant glass transition in colloidal disks [12]. Reentrant isotropic-nematic phase behavior has been found in polymer-depleted amyloid fibrils as a result of modifications in the Derjaguin-Landau-Verwey-Overbeek (DLVO) potential [7]. In biological systems such as DNA and proteins, reentrant condensation as induced by multivalent counterions has also been reported $[10,14]$.

The evolution of interactions between charged colloids in the presence of multivalent counterions cannot be explained by the famous Debye-Hückel (DH) theory [15]. According to this theory, the counterions form a cloud around the colloidal particles and the resultant colloid-counterion complexes interact via a screened Coulomb potential [15]. However, the validity of the theory is restricted to monovalent counterions only. Although the presence of multivalent counterions follows the Hofmesiter series, it leads to many alluring phenomena (e.g., attraction between like charges, charge reversal, etc.), which are not predictable from linear DH or even nonlinear Poisson-Boltzmann (PB) equations [16]. The central idea behind the emergence of all these counterintuitive effects is believed to be the correlations persisting among the adsorbed multivalent ions due to strong interactions with the macroion surface and with each other. This can give rise to overscreening of the colloids and concomitantly charge inversion, unlike the

*Corresponding author: vkaswal@barc.gov.in screening accounted for in DH theory, compensating only a part of the colloid charge [16-18]. However, the consequences of these correlations on the interplay of interparticle interactions are far from clear.

Charge inversion effects are of special interest in biological systems where many natural processes such as the delivery of genes to living cells for gene therapy are driven by charge inversion [16,19]. All these systems usually show a complex structure as well as complex charge distribution where the site-specific adsorption of the ions and hence the correlations among them are expected [10,14,20,21]. Unlike biological systems, in this Rapid Communication, we show a systematic reentrant transition in uniformly charged silica nanoparticles in the presence of multivalent counterions. Anionic silica nanoparticles undergo a transformation from one phase (individual) to two phases (nanoparticle aggregation) and return to one phase upon adding multivalent (triand tetra-) counterions. Optical transmission and dynamic light scattering (DLS) measurements were used to construct the phase behavior. Small-angle neutron scattering (SANS) provides modifications of the interactions prior to and after the two-phase formation while zeta potential measurements demonstrate a charge reversal in the double layer around the nanoparticles at higher salt concentrations.

Electrostatically stabilized anionic silica nanoparticles (HS40) and various salts $\left(\mathrm{NaCl}, \mathrm{MgCl}_{2}, \mathrm{AlCl}_{3}\right.$, and $\left.\mathrm{ZrCl}_{4}\right)$ providing different valence counterions were purchased from Sigma-Aldrich. All samples were prepared in water and no buffer was used to control the $p \mathrm{H}$ in order to observe solely the effect of the addition of salts. The nanoparticles were found to have a mean size of $18 \mathrm{~nm}$ as measured by SANS, a hydrodynamic size of $21 \mathrm{~nm}$ as obtained by DLS, and a measured zeta potential of about $-60 \mathrm{mV}$ [22]. Figure 1(a) shows the phase behavior of a $1 \mathrm{wt} \%$ nanoparticle solution as a function of different salt concentrations. It was determined by measuring the optical transmission of the solutions at a wavelength of $633 \mathrm{~nm}$. The relative transmissions of the samples were obtained by dividing their transmissions with that of the pure $1 \mathrm{wt} \%$ silica nanoparticle system. For monovalent counterions $\left(\mathrm{Na}^{+}\right)$, the transmission remains constant over a wide range of concentrations and systematically decreases at higher salt concentrations. On the other hand, for higher valent counterions $\left(\mathrm{Al}^{3+}\right.$ and $\left.\mathrm{Zr}^{4+}\right)$, the transmission shows an 


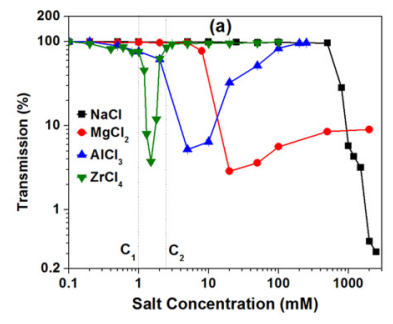

(b)
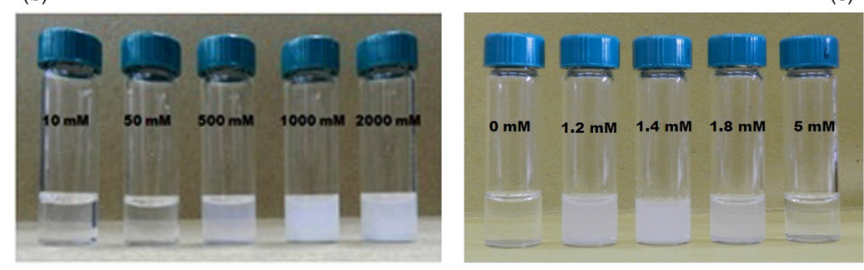

FIG. 1. (a) The relative transmission (\%) of light through $1 \mathrm{w} t \%$ $\mathrm{HS} 40$ silica nanoparticles with varying salt $\left(\mathrm{NaCl}, \mathrm{MgCl}_{2}, \mathrm{AlCl}_{3}\right.$, and $\mathrm{ZrCl}_{4}$ ) concentrations. The physical states of the system with varying salts (b) $\mathrm{NaCl}$ and (c) $\mathrm{ZrCl}_{4}$ are shown.

interesting reentrant behavior where it reduces from $100 \%$ to nearly zero $(<5 \%)$ and then returns to its initial value $(100 \%)$ with increasing salt concentration. The transmission of light through such a system usually reflects the evolution of the structures where the formation of larger (smaller) composites scatters more (less) light and henceforth decrease (increase) in transmission [9]. This behavior is also evident from the physical appearance (turbidity) of the samples [Figs. 1(b) and $1(\mathrm{c})]$. Therefore, the reduction in the transmission beyond a critical salt concentration $C_{1}$ suggests the transformation of a stable one-phase nanoparticle system into an aggregated state (two-phase system) for increasing counterion concentration, in accordance with DH theory. However, contrary to the expectations of DH theory, the nanoparticle aggregates (two-phase system) again transform to stabilized individual nanoparticles (one-phase system) at higher multivalent counterion $\left(\mathrm{Al}^{3+}\right.$ and $\mathrm{Zr}^{4+}$ ) concentrations [Fig. 1(c)]. Unlike the case of monovalent counterions $\left(\mathrm{Na}^{+}\right)$, the formation of a two-phase system in the presence of multivalent counterions takes place in between the two critical salt concentrations $C_{1}$ and $C_{2}$, where both of these shift to lower values for the higher valent counterions. For divalent counterions $\left(\mathrm{Mg}^{2+}\right)$, the system does not return to its original transmission (100\%) but exhibits a slow tendency for attaining increasing transmission values of up to about $10 \%$ at $2 M$. Figure 2(a) depicts the complete phase diagram of the nanoparticle system in the presence of the tetravalent counterion $\mathrm{Zr}^{4+}$, as a function of the nanoparticle as well as salt concentrations.

The phase diagram can be divided into three regions where the phase boundaries obey an almost linear relationship with the nanoparticle concentrations. We have also examined the system for varying concentrations of the nanoparticles and with different salts $\left(\mathrm{CsCl}, \mathrm{CaCl}_{2}, \mathrm{YCl}_{3}\right)$ (Fig. 3). The essential features of the phase behaviors are found to be dependent only on the valency of the counterions, suggesting the universal nature of this reentrant behavior for multivalent counterions. The nature of system evolution with varying salt has been ascertained by DLS experiments. DLS measurements were carried out on the nanoparticle size and zeta potential
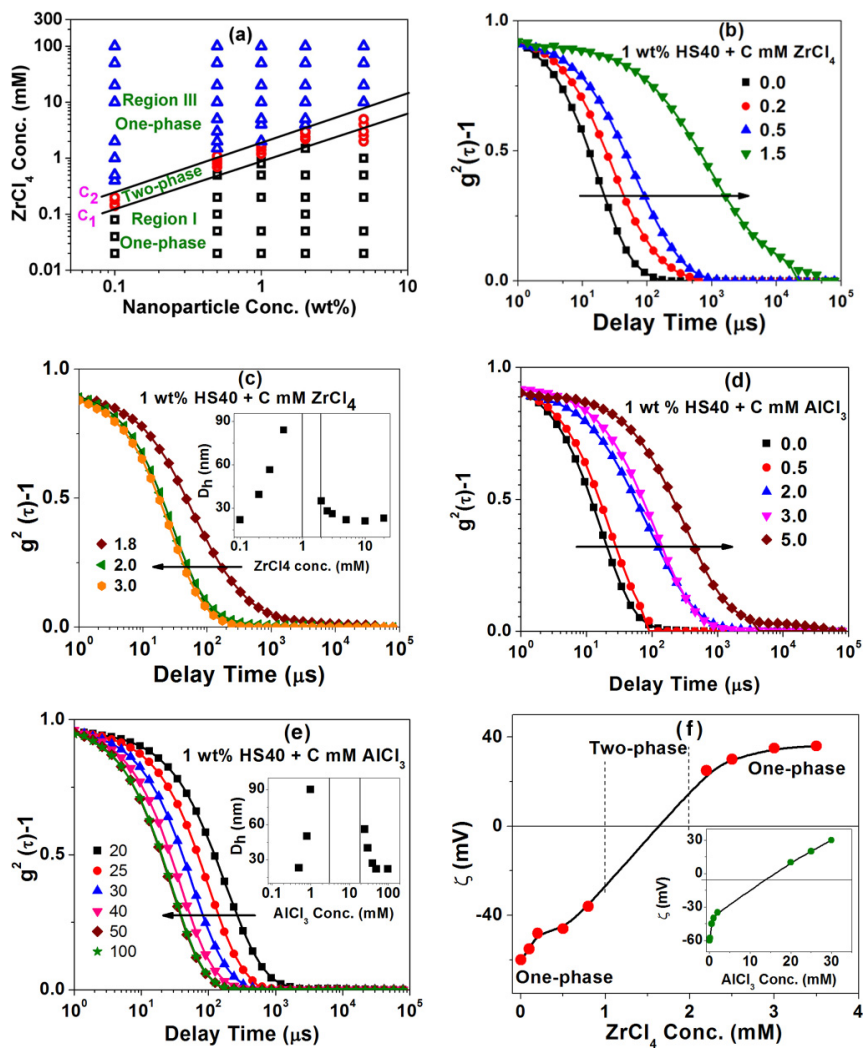

FIG. 2. (a) Phase behavior of silica nanoparticle solutions as a function of nanoparticle and salt $\left(\mathrm{ZrCl}_{4}\right)$ concentrations. Squares, circles, and triangles represent the system in the one-phase, two-phase, and again in the one-phase region, respectively. The autocorrelation functions in DLS of $1 \mathrm{wt} \%$ nanoparticles as a function of $\mathrm{ZrCl}_{4}$ (b) on approaching the two-phase region and (c) returning to the one-phase region. The data for $\mathrm{AlCl}_{3}$ corresponding to (b) and (c) are shown in (d) and (e), respectively. The insets of (c) and (e) show the variations of calculated effective hydrodynamic size $\left(D_{h}\right)$. (f) Variation in the zeta potential $(\zeta)$ of a nanoparticle system with increasing salt $\left[\mathrm{ZrCl}_{4}\right.$ and $\mathrm{AlCl}_{3}$ (inset)] concentrations.

analyzer SZ-100 (Horiba, Japan) utilizing an incident laser light of wavelength $532 \mathrm{~nm}$ and the intensity fluctuations were measured at a scattering angle of $173^{\circ}$. The corresponding intensity autocorrelation functions of $1 \mathrm{wt} \%$ nanoparticles with varying $\mathrm{ZrCl}_{4}$ concentrations are shown in Figs. 2(b) and 2(c). The addition of salt up to concentration $C_{1}$ leads to a shift in the diffusive relaxation to a longer relaxation time (as indicated by the arrow) as the system approaches from one phase to two phases [Fig. 2(b)]. The data show only one relaxation mode and hence a cumulant method has been used for the analysis $[23,24]$. The calculated values of $D_{h}$ using the Stokes-Einstein relation are shown in the inset of Fig. 2(c). The increase in effective $D_{h}$ may arise because of two contributions: (i) an enhanced attraction between the nanoparticles and/or (ii) the formation of larger nanoparticle aggregates. To unfold these two contributions, the DLS experiments were further performed after diluting the systems by a factor of 10 . The dilution is supposed to suppress the apparent increase in the effective hydrodynamic size as caused by attractive interactions (if any). On dilution, the effective sizes of the system for salt concentrations below 

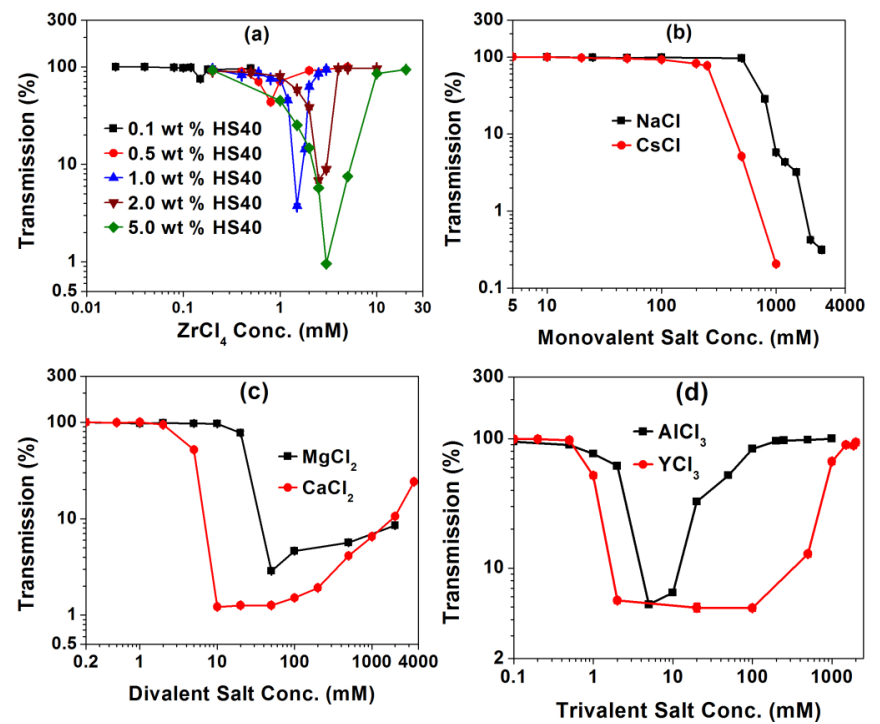

FIG. 3. (a) The relative transmission (\%) of light through varying concentrations of HS40 silica nanoparticles as a function of $\mathrm{ZrCl}_{4}$ concentration. The relative transmissions of 1 wt \% HS40 nanoparticles are depicted with varying (b) monovalent $(\mathrm{NaCl}$ and $\mathrm{CsCl})$, (c) divalent $\left(\mathrm{MgCl}_{2}\right.$ and $\left.\mathrm{CaCl}_{2}\right)$, and (d) trivalent $\left(\mathrm{AlCl}_{3}\right.$ and $\left.\mathrm{YCl}_{3}\right)$ salts.

$C_{1}$ reduce to those of a dilute pure nanoparticle system, indicating that the system is solely governed by the evolution of net attraction in this region of phase behavior. In the two-phase region of the phase diagram [Fig. 2(a)], the system shows a very large effective hydrodynamic size representing the nanoparticle aggregates. On further increasing the salt concentration beyond $C_{2}$, autocorrelation functions [Fig. 2(c)] shift back towards that of pure nanoparticles, suggesting the devolution of the net attraction in the system and hence a reentrant phase behavior. The autocorrelation functions in DLS of $1 \mathrm{wt} \%$ nanoparticles with varying concentrations of $\mathrm{AlCl}_{3}$ also show similar features to those of $\mathrm{ZrCl}_{4}$ on approaching a two-phase region [Fig. 2(d)] and returning to the one-phase region [Fig. 2(e)].

Figure 2(f) and its inset show the measured zeta potential of $1 \mathrm{wt} \%$ silica nanoparticles with the addition of varying concentrations of $\mathrm{ZrCl}_{4}$ and $\mathrm{AlCl}_{3}$, respectively. The zeta potential changes sign for salt concentrations greater than $C_{2}$, implying an inversion of the nanoparticle charge. The magnitude of the inverted zeta potential is observed to be more than $+30 \mathrm{mV}$, sufficient for the formation of a quite stable system. However, it is significantly less than the original value $(-60 \mathrm{mV})$ and that of the expectations of the giant charge inversion (more than $100 \%$ of original charge) theory $[17,25]$. The smaller value could be because of the geometry and/or different surface charge densities of the particles under investigation. It may be added that reliable zeta potential measurements could not be made in the two-phase region due to turbidity in the samples.

Observations of the reentrant phase transition so far have been accomplished by macroscopic techniques. However, to quantify the interplay of the interactions directing the reentrant phase behavior, SANS studies were performed. Measurements were carried out at the SANS-I facility at the
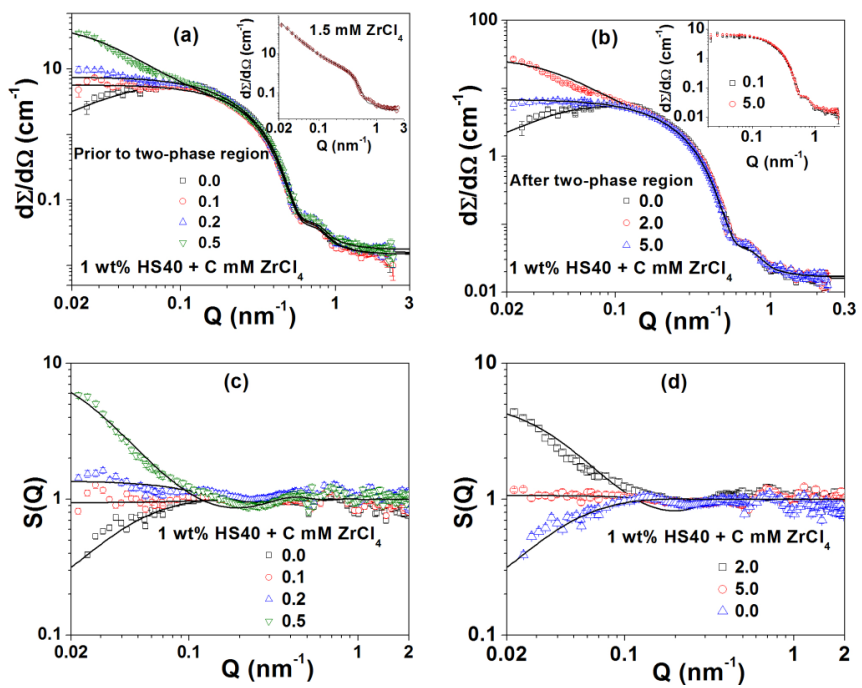

FIG. 4. The SANS data of 1 wt $\%$ nanoparticle systems with varying $\mathrm{ZrCl}_{4}$ concentrations, (a) prior and (b) after the two-phase formation. The corresponding structure factors are shown in (c) and (d). The inset of (a) shows the data in the two-phase region and (b) shows the comparison of data corresponding to regions I and III of the phase behavior.

Paul Scherrer Institut, Switzerland, over a wide scattering vector $[Q=4 \pi \sin (\theta / 2) / \lambda$, with neutron wavelength $\lambda$ and scattering angle $\theta$ ] range $\left(0.02-2.5 \mathrm{~nm}^{-1}\right)$ [26]. In SANS, the measured coherent differential scattering cross section per unit volume $[d \Sigma / d \Omega(Q)]$ is related to the form factor $P(Q)$ and structure factor $S(Q)$ by the relation $\frac{d \Sigma}{d \Omega}(Q)=$ $\varphi V\left(\rho_{p}-\rho_{s}\right)^{2} P(Q) S(Q)$, where $\varphi$ is the volume fraction and $V$ is the volume of the particles. $\rho_{p}$ and $\rho_{s}$ are scattering length densities of the particles and solvent, respectively. $P(Q)$ decides the geometry of the particles while $S(Q)$ originates from the interparticle interactions [27]. Figures 4(a) and 4(b) show the SANS data of a $1 \mathrm{wt} \%$ silica nanoparticle system with varying concentrations of $\mathrm{ZrCl}_{4}$. The SANS data are divided into two sets, prior to $(<1 \mathrm{mM})$ and after $(\geqslant 2 \mathrm{mM})$ the two-phase formation in the phase behavior [Figs. 1(a) and 2(a)]. In the first data set [Fig. 4(a)], there is a systematic scattering buildup in the low $Q$ region without substantial changes in the intermediate and high $Q$ regions. The increase in the scattering is attributed to an enhanced attraction, as also discussed for the DLS experiments. In the second data set [Fig. 4(b)], this low $Q$ scattering buildup is suppressed with an increase in the salt concentration as the two-phase system returns to a one-phase system. The SANS data of nanoparticles at $5 \mathrm{mM}$ salt concentration almost matches that at $0.1 \mathrm{mM}$, evidencing the reentrance of the system to one phase [inset of Fig. 4(b)]. Figures 4(c) and 4(d) show the structure factor $S(Q)$ corresponding to the SANS data [Figs. 4(a) and 4(b)] as calculated by dividing the experimental data with that of the dilute nanoparticle system ( $\left.1 \mathrm{wt} \% \mathrm{HS} 40+0.1 \mathrm{mM} \mathrm{ZrCl}_{4}\right)$. The $S(Q)$ have been fitted by using the two-Yukawa potential as given by $\frac{V_{2 Y}(r)}{k_{B} T}=-K_{1} \frac{\exp \left[-Z_{1}(r / \sigma-1)\right]}{r / \sigma}+K_{2} \frac{\exp \left[-Z_{2}(r / \sigma-1)\right]}{r / \sigma}$, where the interparticle distance is represented by $r$ and $\sigma$ is the hard sphere diameter of the particle [28]. Since the interactions governing the present system are primarily electrostatic in 

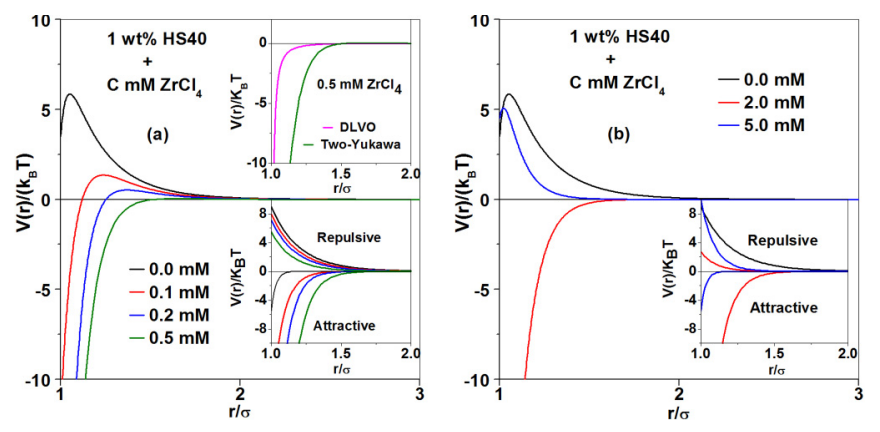

FIG. 5. The variation of the total interaction potential along with attractive and repulsive parts (insets) between nanoparticles as a function of $\mathrm{ZrCl}_{4}$ approaching from (a) one phase to two phase and (b) two phase to one phase. The upper inset of (a) shows the comparison of the calculated DLVO potential and fitted two-Yukawa potential at $0.5 \mathrm{mM}$ of $\mathrm{ZrCl}_{4}$.

nature, hence the choice of such a potential provides the most suitable representation of the system. The first term represents the interparticle attraction, while the second one accounts for the interparticle repulsion. The potential contains four parameters $K_{i}$ and $Z_{i}(i=1,2)$ which represent the strength and range $(\sigma / Z)$ of the respective parts of the potentials. The interactions and stability of the charged nanoparticles are usually described by DLVO theory, which combines the electrostatic repulsion with the van der Waals attraction. The presence of salt ions suppresses the repulsion between charged nanoparticles, yielding a net attraction in the DLVO potential [29]. In the first data set [Fig. 4(a) and 4(c)], such a salt-induced reduction in electrostatic repulsion has been taken into account by appropriately modifying the parameters $K_{2}$ and $Z_{2}$ as per DH theory, which are related to the effective charge and Debye length (ionic strength) of the electrostatic repulsion, respectively [30]. This repulsive potential along with the van der Waals attraction does not fit the data. The additional counterion-induced attraction beyond the $\mathrm{DH}$ theory needs to be considered, where the parameters $K_{1}$ and $Z_{1}$ are used as fitting parameters [23]. The fitted potentials along with attractive and repulsive components (insets) are shown in Fig. 5.

Our analysis shows that salt ions induce an attraction more than can be simply accounted for by the DLVO potential. For salt concentrations $\geqslant 0.2 \mathrm{mM}$ in the first data set [Fig. 4(a)], the electrostatic repulsion becomes negligible and the system interaction is expected to be predominantly dictated by a van der Waals attraction, as per the DLVO theory. In this case, a van der Waals attraction is insufficient to cause an immediate aggregation of the particles, as observed in the phase behavior. This is because the strength of the van der Waals interaction is less than the average thermal kinetic energy $\left(1.5 k_{B} T\right)$ at an average distance between the nanoparticles. This condition, along with the necessity to explain the observed SANS data, required an additional attraction beyond DH theory between the nanoparticles caused by salt ions. The comparison of the fitted two-Yukawa potential with the DLVO potential at $0.5 \mathrm{mM}$ of $\mathrm{ZrCl}_{4}$ is depicted in the upper inset of Fig. 5(a). The screened Coulomb potential is calculated from the Debye length and charge on the nanoparticles, and the van der
Waals attraction has been computed using a Hamaker constant $H=0.5 \times 10^{-20} \mathrm{~J}$ for silica. It can be inferred that the parameters of the fitted two-Yukawa potential are significantly enhanced as compared to the DLVO potential.

Upon further increasing the salt concentration $(\geqslant 1 \mathrm{mM})$, the salt-induced attraction between the nanoparticles becomes sufficiently strong to lead the particle aggregation (twophase system). The data in the two-phase region $[1.5 \mathrm{mM}$ $\mathrm{ZrCl}_{4}$ shown in the inset of Fig. 4(a)] cannot be fitted by considering the attractive interaction alone, and as the phase behavior suggests, the data have been analyzed by considering the presence of nanoparticle aggregates. The nanoparticle aggregates are found to be mass fractal in nature with a fractal dimension of about 2.5. The data in the second set [Fig. 4(b)], showing a systematic suppression of the scattering intensity, have also been analyzed using $S(Q)$ calculated for the two-Yukawa potential. The potentials plotted in Fig. 5(b) show a systematic decrease in magnitude as well as in range with increasing salt concentration in region III of the phase behavior.

In order to understand the role of the valency of the counterions causing differences in the phase behavior (Fig. 1), the SANS data of the $1 \mathrm{wt} \%$ nanoparticles in the presence of varying $\mathrm{AlCl}_{3}$ have also been measured and analyzed in the same manner as described above (see the Supplemental Material [23]). A comparison of the evolution of the attraction caused by $\mathrm{Al}^{3+}$ and $\mathrm{Zr}^{4+}$ suggests that higher valent counterions $\left(\mathrm{Zr}^{4+}\right.$ rather than $\left.\mathrm{Al}^{3+}\right)$ are capable of inducing a more efficient attraction between the nanoparticles.

The possibility of multivalent counterions inducing shortrange attractions between like charged particles has been reported theoretically where either the positional correlations of the counterions or the charge fluctuations are supposed to be responsible for the origin of the attraction [31,32]. It has been shown that the onset of a counterion-induced attraction between like charged colloids depends on the characteristic radius related to both the ion radius and its valency [32]. As a result, the strength as well as the range of the attraction potential is found to be higher for the higher-valent counterions. A counterion-driven attraction between like charged DNA strands and polyelectrolytes has already been observed $[33,34]$.

The phase behavior thus can be understood in terms of the interplay of the long-range electrostatic repulsion between the nanoparticles and the ion-induced short-range attraction. It may be noted that the ion-induced attraction plays an important role only in the case of multivalent counterions, while for monovalent counterions it remains quite small (if at all). At low concentrations of multivalent counterions, electrostatic repulsion between nanoparticles dominates over the ion-induced attraction and the system remains in one phase. Counterion-induced attraction starts dominating with an increase in salt concentration, as the repulsion is significantly reduced by the increased ionic strength in the system, leading to the two-phase formation. On further addition of the salt, the charge of the nanoparticles reverses its sign after a critical salt concentration $\left(C_{2}\right)$ [Fig. 2(f)]. Beyond $C_{2}$, the monovalent $\mathrm{Cl}^{-}$ions play the role of counterions which are not able to induce a sufficient attraction to sustain a two-phase region. It may be further pointed out that all other forces such as 
hydrophobic interactions, solvation forces, and thermal and entropic contributions were neglected due to their minimal role in the interaction potential of the present system.

The system behavior in the reentrant state [region III of Fig. 2(a)] demonstrates the unexpectedly enhanced stability against the counterions in spite of the low zeta potential values compare to the original system. As can be seen in Fig. 1(a), the addition of about $2 \mathrm{M} \mathrm{Na}^{+}$in $1 \mathrm{wt} \%$ nanoparticles leads to an almost instant aggregation of the nanoparticles. Contrary to this, the system in region III (e.g., $1 \mathrm{wt} \% \mathrm{HS} 40+10 \mathrm{mM}$ $\mathrm{ZrCl}_{4}$ ) does not become turbid on the addition of $\mathrm{NaCl}$ even up to the solubility limit. Even the addition of more effective counterions as per the Hofmeister series $\left(\mathrm{I}^{-}>\mathrm{Br}^{-}>\mathrm{Cl}^{-}\right)$ up to $2 M$ does not show any signature of nanoparticle aggregation (see the Supplemental Material [23]).This shows that the presence of multivalent counterions is able to tune the double layer formed around the nanoparticles to provide them such a high stability. These observations are beyond the scope of the well-established mean-field theories and extensive investigations are required for a complete understanding.

To conclude, we have demonstrated multivalent counteriondriven reentrant phase transitions in a model charged colloidal system. The phase boundaries are shown to be governed by mainly the ion valency which offers control and tunability of the system stability. The phase behavior has been explained in terms of the cumulative effects of salt-induced attraction and electrostatic repulsion between nanoparticles which dictates the system behavior in different salt concentration regimes.
[1] H. Mundoor, B. Senyuk, and I. I. Smalyukh, Science 352, 69 (2016).

[2] V. D. Nguyen, S. Faber, Z. Hu, G. H. Wegdam, and P. Schall, Nat. Commun. 4, 1584 (2013).

[3] A. Yethiraj, Soft Matter 3, 1099 (2007).

[4] F. Leoni and Y. Shokef, Phys. Rev. Lett. 118, 218002 (2017).

[5] A. K. Arora and B. V. R. Tata, Adv. Colloid Interface Sci. 78, 49 (1998).

[6] F. Bomboi, F. Romano, M. Leo, J. Fernandez-Castanon, R. Cerbino, T. Bellini, F. Bordi, P. Filetici, and F. Sciortino, Nat. Commun. 7, 13191 (2016).

[7] J. Zhao, C. Li, and R. Mezzenga, J. Phys.: Condens. Matter 26, 464112 (2014).

[8] B. Koh and W. Cheng, Langmuir 30, 10899 (2014).

[9] S. Kumar, V. K. Aswal, and J. Kohlbrecher, Langmuir 32, 1450 (2016).

[10] F. Zhang, M. W. A. Skoda, R. M. J. Jacobs, S. Zorn, R. A. Martin, C. M. Martin, G. F. Clark, S. Weggler, A. Hildebrandt, O. Kohlbacher, and F. Schreiber, Phys. Rev. Lett. 101, 148101 (2008).

[11] J. Möller, S. Grobelny, J. Schulze, S. Bieder, A. Steffen, M. Erlkamp, M. Paulus, M. Tolan, and R. Winter, Phys. Rev. Lett. 112, 028101 (2014).

[12] C. E. Sing, J. W. Zwanikken, and M. O. de la Cruz, Macromolecules 46, 5053 (2013).

[13] A. K. Atmuri, G. A. Peklaris, S. Kishore, and S. R. Bhatia, Soft Matter 8, 8965 (2012).

[14] T. T. Nguyen, I. Rouzina, and B. I. Shklovskii, J. Chem. Phys. 112, 2562 (2000).

[15] P. Debye and E. Hückel, Physikalische Zeitschrift 24, 185 (1923).

[16] A. Yu. Grosberg, T. T. Nguyen, and B. I. Shklovskii, Rev. Mod. Phys. 74, 329 (2002).

[17] T. T. Nguyen, A. Yu. Grosberg, and B. I. Shklovskii, Phys. Rev. Lett. 85, 1568 (2000).
[18] A. Diehl and Y. Levin, J. Chem. Phys. 121, 12100 (2004).

[19] A. Kubíčková, T. Křížek, P. Coufal, M. Vazdar, E. Wernersson, J. Heyda, and P. Jungwirth, Phys. Rev. Lett. 108, 186101 (2012).

[20] Y. Levin, Rep. Prog. Phys. 65, 1577 (2002).

[21] K. Besteman, K. V. Eijk, and S. G. Lemay, Nat. Phys. 3, 641 (2007).

[22] I. Yadav, V. K. Aswal, and J. Kohlbrecher, Phys. Rev. E 93, 052601 (2016).

[23] See Supplemental Material at http://link.aps.org/supplemental/ 10.1103/PhysRevE.96.060602 for DLS and SANS Analyses, SANS data of reentrant phase behavior of silica nanoparticles with $\mathrm{AlCl}_{3}$, and stability of reentrant phase against the monovalent salts.

[24] P. A. Hassan, S. Rana, and G. Verma, Langmuir 31, 3 (2015).

[25] M. Tanaka and A. Y. Grosberg, J. Chem. Phys. 115, 567 (2001).

[26] J. Kohlbrecher and W. Wagner, J. Appl. Crystallogr. 33, 804 (2000).

[27] D. I. Svergun and M. H. J. Koch, Rep. Prog. Phys. 66, 1735 (2003).

[28] Y. Liu, W.-R. Chen, and S.-H. Chen, J. Chem. Phys. 122, 044507 (2005).

[29] B. V. Derjaguin and L. Landau, Acta PhysicoChim URSS 14, 633 (1941).

[30] A. J. Chinchalikar, V. K. Aswal, J. Kohlbrecher, and A. G. Wagh, Phys. Rev. E 87, 062708 (2013).

[31] B.-Y. Ha and A. J. Liu, Phys. Rev. Lett. 79, 1289 (1997).

[32] N. Gronbech-Jensen, R. J. Mashl, R. F. Bruinsma, and W. M. Gelbart, Phys. Rev. Lett. 78, 2477 (1997).

[33] T. E. Angelini, H. Liang, W. Wriggers, and G. C. L. Wong, Proc. Natl. Acad. Sci. USA 100, 8634 (2003).

[34] X. Qiu, K. Andresen, L. W. Kwok, J. S. Lamb, H. Y. Park, and L. Pollack, Phys. Rev. Lett. 99, 038104 (2007). 